\title{
Virtually anything can change
}

\author{
Coronavirus-imposed restrictions are putting great strain on academia and the research community but there may \\ be a silver lining in all the commotion.
}

A cademia has been forced to radically change its normal operations in the wake of the coronavirus crisis. Research laboratories have been closed or had their activities limited, scientific conferences have gone virtual and universities have moved their lectures online. Now that some universities are switching to a new normality, we are starting to see how the restrictions of the past months may shape academia in the longer term.

Time away from the workplace has given some the opportunity to focus more on finishing research papers, grant applications and student supervision. Yet, prolonged closure of facilities or new shift-work restrictions mean that research work will now likely take longer to complete. Those undertaking field research are having to reconsider how they go about it safely or whether it is even possible. These impacts could be costly in terms of funding and career progression.

Resuming research activities provides an opportunity to improve lab operations. We can think about what we did before and why, and change it to benefit research endeavours. For instance, efficiency, transparency and reproducibility can be aided by promoting better data sharing practices between group members and collaborators.

As we are all now keenly aware, travel restrictions have meant that most conferences this year have migrated online. This is a welcome change for many, as virtual meetings can be more inclusive and have a lower carbon footprint and less environmental impact. In a Comment in this issue, Michael Saliba at the University of Stuttgart shares his experience in organizing a virtual meeting, which could help others to set up their own.

Virtual meetings save on travel time and can allow better time management through attendance only at sessions of interest. This presents an opportunity to indulge in conferences on subjects distant from one's immediate area of research, which may inspire more interdisciplinary and intersectoral research.

However, virtual meetings also have drawbacks. Personal interactions are one aspect that is particularly missed in virtual conferences. Conference breaks are traditionally an opportunity to network and showcase one's research in more informal ways. Serendipitous interactions can result in unexpected collaborations or new research ideas. This can help especially early-stage researchers. Yet these fruitful encounters may mainly favour privileged groups and reinforce existing networks. For every story of the benefit of a chance encounter, how many stories are there of people being dismissed or ignored or unable to take the chance?

Reorienting to virtual meetings provides a moment to consider why we hold conferences, what we want to get from them, and how they reflect the values and spirit we hold dear. We do not have to replicate the in-person experience online - we can build a new one that brings more benefits for more people, that seeks to foster new and more inclusive ways of interacting with one another.

We are still figuring out what works well and what does not in online meetings. There is a lot of room for improving and experimenting. Hybrid approaches that combine in-person with virtual features could make the best use of both formats. What we learn from these experiments could offer the community a unique chance to move towards a different conference system that does not shore up ways of operating that do not actively cultivate diversity and inclusion.

Education is also being impacted by moving online. Teachers and students have had to adapt to new ways of teaching, learning and developing research projects. Key to the success of online lectures is the relationship between students and educators. Technology can break down some barriers: it can allow for a more diversified classroom and intimate connections that marginalized students, in particular, can benefit from ${ }^{1}$.

As social distancing measures are likely to stay in place over the next academic year, even if some face-to-face classes will be resumed, online teaching and training will continue in the short term. This could come at a cost. For instance, it can be hard to remotely develop soft skills around research culture and work practice in students. Supervisors may struggle to keep an eye on students and step in at the right time if things go wrong. The acquisition of practical skills can be hard through virtual experiments. If universities shift some classes online for the long term, they should seek to balance virtual and in-person meetings with supervisors and pair with local universities to offer in-person mentorship for students that are not in the same city as their university, where safe to do so. Campus experiences, that add value and contribute to personal growth, will also not be straightforward to replicate. These drawbacks and uncertainties may cause students to defer their admission to future semesters, potentially creating gaps in the education of next-generation researchers.

On the other hand, going to university and living in student accommodation is not affordable or feasible for some students. For them, online and distance learning represents an opportunity to get a degree. University reopening plans should consider an integrated approach to teaching and learning that blends virtual with in-person offers as a way to foster diversity, equity and inclusion in higher education.

Coronavirus restrictions have forced academia to rapidly change its normal ways of working with little time to figure out which responses are the best. While it is too early to know whether some of these changes will become permanent, we should evaluate the previous and current systems to shape the new normal with a view to improving the way research is done and promoting a more inclusive and fairer community.

We at Nature Energy believe that engaging all parties - researchers, educators, students - in a constructive dialogue could lay the groundwork for far-reaching reforms. We would like to facilitate this where possible. E-mail us at natureenergy@nature.com to let us know what steps you think would actively nurture diversity, equity and inclusion in higher education and across academic ranks and what actions would bring in new voices to the scientific debate at conferences. We will publish the most insightful comments.

Published online: 17 July 2020

https://doi.org/10.1038/s41560-020-0663-0

\footnotetext{
References

1. Roll, K. \& Ventresca, M. Lecturer and student relationships matter even more online than on campus. The Guardian https://www.theguardian.com/education/2020/jun/08/ lecturer-and-student-relationships-matter-even-more-onlinethan-on-campus (2020).
} 